\title{
Plasma microRNAs are promising novel biomarkers for the early detection of Toxoplasma gondii infection
}

Boyin Jia ${ }^{\dagger}$, Zhiguang Chang ${ }^{\dagger}$, Xiaoyan Wei, Huijun Lu, Jigang Yin, Ning Jiang ${ }^{*}$ and Qijun Chen*

\begin{abstract}
Background: MicroRNAs (miRNAs) have been shown to be present in plasma, which are remarkably stable, and have been suggested as disease biomarkers. Toxoplasma gondii (T. gondii) is a protozoan parasite that is infective to a wide range of animals and human beings. Previous studies have found that the parasite generated a large number of miRNAs during proliferation and it is known that the spectrum of miRNA expression in the infected hosts is pathogen-specific. To date, there are no reports regarding the application of microRNAs as biomarkers for the early detection of T. gondii infection.

Methods: In this study, we investigated the expression patterns of 414 murine miRNAs and tested their expression levels in the plasma after T. gondii infection by real-time PCR, with an ultimate purpose of identifying infection-related miRNAs. Three miRNAs in particular, exhibiting prominently elevated expressions, were further validated in a large number of infected mice. The Toxoplasma infection-specific miRNAs were confirmed by comparing their expression levels with those of mice infected with Plasmodium berghei, P. yoelii, P. chabaudi, Cryptosporidium parvum, Mouse hepatitis virus, and Staphylococcus aureus.
\end{abstract}

Results: Among the 414 miRNA candidates identified by a real-time PCR array, 71 were found to be up-regulated in the plasma of T. gondii infected mice. Three of those miRNAs (mmu-miR-712-3p, mmu-miR-511-5p and mmu-miR-217-5p) were prominently expressed in mice infected by both the RH and ME49 strains of T. gondii. Additionally, the elevated expression of these miRNAs was Toxoplasma-specific.

Conclusions: The levels of the three miRNAs, mmu-miR-712-3p, mmu-miR-511-5p and mmu-miR-217-5p miRNAs, were found specifically up-regulated in plasma of mice after T. gondii infection.

Keywords: Toxoplasma gondii, Plasma, MicroRNAs, Biomarker, Real-time PCR

\section{Background}

T. gondii is an obligate intracellular parasite that causes diverse pathological effects in humans and other warmblooded vertebrates $[1,2]$. Toxoplasmosis, caused by T. gondii, is a worldwide parasitic disease that is widespread in Asia, Africa, South America, and Europe [3-5]. The parasite can cause severe disease in immunocompromised patients as well as in pregnant mothers [6,7]. Toxoplasmosis may cause serious consequences in new-born babies, such as hearing and sight impairment and neurological symptoms

\footnotetext{
* Correspondence: jiangning@jlu.edu.cn; cqj@jlu.edu.cn

${ }^{\dagger}$ Equal contributors

Key Laboratory of Zoonosis, Institute of Zoonosis/College of Veterinary Medicine, Jilin University, Xi An Da Lu 5333, Changchun 130062, China
}

[8]. Early treatment of pregnant women could reduce the incidence of sequelae in infected infants [9]. Detection of early-stage toxoplasmosis is a key measure in reducing toxoplasmosis-related health damage.

Serological studies are currently the most common diagnostic method for acute infections; however, they have several disadvantages. Parasite-specific antibodies are not normally present during the early stages of infection, particularly in immunosuppressed patients and in pregnant patients $[10,11]$. Other detection methods include PCR assays that are sensitive and fast and can detect parasites from different samples such as amniotic fluid and tissues [12,13]. However, the detection of T. gondii in amniotic fluid remains unsatisfactory because 
of false negative and false positive findings [14]. Therefore, an ideal method with high specificity and high sensitivity for the early stage diagnosis of $T$. gondii infection is urgently needed.

MicroRNAs (miRNAs) are 21-25 nucleotide noncoding RNA molecules that play important roles in physiological and pathological processes [15]. T. gondii-derived miRNAs have been identified in different parasite strains, which will facilitate the dissection of the parasite biology in different biological environments [16,17]. In addition, the miRNA expression of the host could be affected by the invasion of T. gondii [18]. Xiao et al. reported that miR-132 was up-regulated in T. gondii infected mice and was associated with changes in dopamine receptor signaling [19]. They also found that miR-30c-1, miR-125b-2, miR-23b-27b-24-1 and miR-17 92 were up-regulated in human macrophages after $T$. gondii infection, which were associated with the anti-apoptosis responses of the host cells [20]. However, the function of both parasite- and host-derived miRNAs in association with parasite infection still needs further study.

Recently, it has been reported that miRNAs are stable enough to be detected in the plasma. In addition, circulating miRNAs from tissues are protected from endogenous RNAse activity [21]. The significance of plasma miRNA levels as biochemical markers for human cancer, such as colorectal cancer, lung cancer, pancreatic cancer, and prostate cancer, has been gradually recognised [22-25]. However, to our knowledge, there are no reports on the characterization of circulating miRNAs in the plasma of patients with $T$. gondii infection.

In this study, a real-time PCR array was applied to measure the levels miRNAs from the plasma of mice infected with T. gondii. We focused on the analysis of three miRNAs, mmu-miR-712-3p, mmu-miR-511-5p and mmu-miR217-5p, which were detected abundantly in mice infected with the RH (Type I) and ME49 (Type II) strains of T. gondii. Quantitative analysis of these miRNAs in a large set of plasma samples from mice showed that mmu-miR-712-3p, $\mathrm{mmu}-\mathrm{miR}-511-5 \mathrm{p}$ and $\mathrm{mmu}-\mathrm{miR}-217-5 \mathrm{p}$ were potentially useful for early stage diagnosis, with a satisfactory degree of sensitivity and specificity.

\section{Methods}

\section{Plasma sample collections}

RH and ME49 strains of T. gondii were routinely kept in the laboratory by cell cultivation. Sixty female BALB/c mice (20-25 g) aged 6 to 8 weeks were randomly divided into 3 groups. For plasma collection from mice infected with T. gondii, 2 groups of BALB/c mice (20 mice per group) were peritoneally infected with $10^{6}$ tachyzoites of RH or ME49 strain per mouse. Infection was confirmed by performing Giemsa staining of peritoneal fluid and by PCR [26,27]. 72 hours after infection, the infected mice were exsanguinated, and the blood was mixed with heparin for the separation of plasma. Blood samples were centrifuged at $1200 \mathrm{~g} / \mathrm{min}$ for 10 minutes at room temperature to remove the blood cells, followed by a second centrifugation at $12000 \mathrm{~g} / \mathrm{min}$ for 10 minutes at $4^{\circ} \mathrm{C}$ to remove cellular components. Plasma samples were stored at $-80^{\circ} \mathrm{C}$ until further processing. Frozen plasma of mice infected with Plasmodium berghei, P. yoelii, P. chabaudi, Cryptosporidium parvum (C. parvum), Mouse hepatitis virus (MHV), and Staphylococcus aureus (S. aureus) were also processed as described above. The permission to work with laboratory animals was obtained from the Ethical Committee of the Institute of Zoonosis, Jilin University, China (Permission number 2008-IZ-20).

\section{RNA isolation}

Total plasma RNA was isolated with the mirVana ${ }^{\text {tw }}$ miRNA Isolation Kit (Ambion, USA) according to the manufacturer's protocol. Briefly, synthetic cel-miR-39 was added to each sample as a spike-in control, and total RNA was purified from $400 \mu \mathrm{l}$ of plasma. RNA was eluted with $100 \mu \mathrm{l}$ of RNase-free water. The concentration of total RNA samples from plasma was quantified by a Nanodrop 2000 (Nanodrop, USA). The range of the result was from 11.9 to $73.7 \mathrm{ng} / \mu \mathrm{l}$.

\section{Real-time quantitative reverse-transcription PCR}

Real-time PCR of 414 mice miRNAs was performed. Total RNA from 3 mice infected with the RH strain of T. gondii and from 3 healthy controls was used. The reversetranscription reaction was initiated with $50 \mathrm{ng}$ of total RNA and was carried out in a volume of $20 \mu \mathrm{l}$ using the miScriptII Reverse Transcription kit (Qiagen, Germany). Cel-miR39 was used as an internal control. MiRNA concentrations were then confirmed by real-time PCR using the miScript SYBR Green PCR Kit (Qiagen, Germany). Universal primers and the miRNA-specific forward primers were provided by Qiagen. Each reaction was performed in a final volume of $20 \mu \mathrm{l}$ containing $2 \mathrm{ng}$ of cDNA, $10 \mu \mathrm{l}$ of $2 \times$ QuantiTect SYBR Green PCR Master Mix, $2 \mu \mathrm{l}$ of $10 \times$ miScript Universal Primer and $2 \mu \mathrm{l}$ of $10 \times$ miScript Primer Assay. The ABI Prism 7900 Sequence Detection System (Ambion, USA) was used for amplification and detection. Differences in miRNAs were normalised to cel-miR-39, determined with the $\Delta \mathrm{Ct}$ method, and reported as $2^{-\Delta \mathrm{Ct}}$.

\section{Statistical analysis}

The quantitative data were analysed with the Mann-Whitney U test. Receiver operating characteristic (ROC) curves were established to examine the accuracy of using miRNAs in early stage detection for diagnosing $T$. gondii infection. A $p$ value less than 0.05 was considered statistically significant. All statistical calculations were performed by the SPSS 19.0 software. 


\section{Results}

Preliminary marker selection on a small set of plasma samples

The aim of this study was to determine whether there are any marked miRNAs with specific expression patterns in mice after a $T$. gondii infection. We performed the initial test using real-time PCR arrays using RNA isolated from $T$. gondii-infected mice and healthy controls. The levels of miRNAs found differed profoundly in the mice before and after infection. In total, $414 \mathrm{miR}$ NAs could be detected by real-time PCR in all 6 samples of the plasma. Of those 414 miRNAs, 71 were significantly up-regulated in the plasma of $T$. gondii-infected mice compared to that of healthy controls. Table 1 shows the 71 miRNAs with their respective detection quantification intensities. Quantification analysis revealed that the top 15 miRNAs differed significantly between the plasma of infected mice and that of normal controls (Figure 1). Of these miRNAs, mmu-miR-712$3 p$, mmu-miR-511-5p and mmu-miR-217-5p were detected with the highest abundance (the average $\mathrm{Ct}$ values were $17.43,27.45$ and 26.08 , respectively). Thus, the three miRNAs were chosen for analysis in further studies.

\section{Independent large-scale validation on plasma samples}

To further evaluate the expression of mmu-miR-712-3p, mmu-miR-511-5p and mmu-miR-217-5p, 60 plasma samples collected from 20 mice infected with RH strain, 20 mice infected with ME49 strain and 20 healthy controls were investigated by real-time PCR. The expression of these miRNAs was found to be significantly upregulated in the plasma of $\mathrm{RH}$ and ME49 strain infected mice compared to that of the healthy controls by the Mann-Whitney U test $(p<0.001$, Figure 2$)$. However, no significant changes of the three miRNAs in the plasma of $\mathrm{RH}$ strain-infected mice compared to that of ME49 strain-infected mice were observed.

ROC curve analyses revealed that the plasma levels of mmu-miR-712-3p, mmu-miR-511-5p and mmu-miR217-5p were also significantly elevated in mice infected with the RH strain of $T$. gondii compared to healthy controls, with ROC curve areas of 0.959 (95\% confidence interval, 0.844 to 0.996 ), 0.995 (95\% CI, 0.902 to 1.000 ) and 0.940 (95\% CI, 0.817 to 0.990 ), respectively (Figure 3A, $\mathrm{B}$ and $\mathrm{C}$ ). The miRNAs were also markedly increased in mice infected with the ME49 strain of T. gondii compared to healthy controls with an AUC (area under the ROC curve) of 0.935 (95\% CI, 0.810 to 0.988), 0.995 (95\% CI, 0.902 to 1.000$)$ and 0.910 (95\% CI, 0.776 to 0.977 ), respectively (Figure $3 \mathrm{D}, \mathrm{E}$ and $\mathrm{F}$ ). In mice infected with $\mathrm{RH}$ strain and compared to control mice, a cut-off value of 4.94 for mmu-miR-712-3p yields a sensitivity of $85 \%$ and a specificity of $100 \%$; at a cut-off value of 4.21 for mmu-miR-511-5p, the optimal sensitivity and specificity were $95 \%$ and $100 \%$, respectively; and at a cut-off value of 2.01 for mmu-miR-217-5p, the sensitivity was $100 \%$ and the specificity was $85 \%$. Meanwhile, for mice infected with ME49 strain and compared to healthy controls, at a cut-off value of 4.34 for mmumiR-712-3p, the sensitivity was $85 \%$ and the specificity was $100 \%$; at a cut-off value of 3.77 for mmu-miR-511$5 \mathrm{p}$, the optimal sensitivity and specificity were $95 \%$ and $100 \%$; respectively; and at a cut-off value of 4.20 for mmu-miR-217-5p, the sensitivity was $80 \%$ and the specificity was $95 \%$.

\section{The elevated expression of miRNAs was specific to $T$. gondii infection}

We further investigated if the three elevated circulating miRNAs in plasma, mmu-miR-712-3p, mmu-miR-511$5 \mathrm{p}$ and mmu-miR-217-5p, were host specific responses to $T$. gondii infection. The expression levels of the three miRNAs in mice infected with $P$. berghei, $P$. yoelii, P. chabaudi, C. parvum, MHV, and S. aureuswere compared to the expression levels of mice infected with $T$. gondii. We surprisingly found that the expression of the three miRNAs was up-regulated only in T. gondii-infected mice and down-regulated in the mice infected by the other agents $(p<0.001$, Figure 4$)$.

\section{Discussion}

Accumulated evidence suggests that small molecules in body fluids are novel markers for the clinical diagnosis of diseases [28-30]. Measurement of plasma miRNA concentrations comprises a very promising field for clinical applications. Tumour-derived miRNAs were first shown to be present in the plasma in 2008 [21]. Lawrie et al. reported that miRNA-21 was a potentially non-invasive diagnostic marker for B cell lymphoma, which was associated with relapse-free survival [31]. McDonald et al. found that miRNA concentrations were higher in the plasma than in sera and that miRNAs were quite stable when frozen for $72 \mathrm{~h}$ [32]. During the last decade, circulating miRNAs as stable blood-based biomarkers have been intensively investigated for the early detection of colorectal cancer, lung cancer, pancreatic cancer, and prostate cancer.

To date, the correlation between plasma miRNA levels and $T$. gondii infection has not been investigated. Here, we found that the detection of circulating miRNAs in the plasma of $T$. gondii-infected mice was feasible and that 15 miRNAs were found to be significantly up-regulated in the plasma of $T$. gondii-infected mice compared to healthy mice, with fold changes $>10$. The expression of the three miRNAs mmu-miR-712-3p, mmumiR-511-5p and mmu-miR-217-5p were further assessed in a large number of mice infected with either $\mathrm{RH}$ or 
Table 1 Seventy-one differentially expressed $(p<0.05)$ circulating miRNAs in infected and control plasma samples

\begin{tabular}{|c|c|c|c|}
\hline MiRNAs & Fold changes & $p$-value & Increased or decreased \\
\hline mmu-miR-712-3p & 80.62 & $9.22 \mathrm{E}-07$ & Increased \\
\hline mmu-mir-511-5p & 47.93 & 0.00133 & Increased \\
\hline mmu-miR-712-5p & 36.60 & 4.99E-06 & Increased \\
\hline mmu-miR-217-5p & 26.45 & 0.00073 & Increased \\
\hline mmu-miR-192-3p & 23.69 & 0.00024 & Increased \\
\hline mmu-miR-129-1-3p & 21.99 & 0.00016 & Increased \\
\hline mmu-miR-721 & 20.19 & 0.00018 & Increased \\
\hline mmu-miR-34a-3p & 18.54 & 0.00065 & Increased \\
\hline mmu-miR-511-3p & 13.76 & 0.00024 & Increased \\
\hline mmu-miR-302a-5p & 13.26 & 0.00470 & Increased \\
\hline mmu-miR-122-5p & 12.02 & 0.00291 & Increased \\
\hline mmu-miR-713 & 11.59 & 0.00043 & Increased \\
\hline mmu-miR-702-3p & 11.59 & 0.00036 & Increased \\
\hline mmu-miR-144-5p & 11.54 & 0.00285 & Increased \\
\hline mmu-miR-711 & 10.50 & 0.00047 & Increased \\
\hline mmu-miR-715 & 9.47 & 0.00049 & Increased \\
\hline mmu-miR-532-3p & 9.08 & 0.00070 & Increased \\
\hline mmu-miR-361-5p & 9.07 & 0.00087 & Increased \\
\hline mmu-miR-543-3p & 8.78 & 0.00096 & Increased \\
\hline mmu-mir-194-1-3p & 8.75 & 0.00213 & Increased \\
\hline mmu-miR-362-3p & 8.32 & 0.00267 & Increased \\
\hline mmu-miR-532-5p & 8.17 & 0.00090 & Increased \\
\hline mmu-miR-10a-5p & 7.85 & 0.02967 & Increased \\
\hline mmu-miR-705 & 7.47 & 0.00212 & Increased \\
\hline mmu-miR-770-5p & 7.31 & 0.00171 & Increased \\
\hline mmu-miR-218-2-3p & 7.27 & 0.00234 & Increased \\
\hline mmu-miR-493-5p & 7.05 & 0.02691 & Increased \\
\hline mmu-miR-762 & 7.03 & 0.00315 & Increased \\
\hline mmu-miR-714 & 7.01 & 0.00216 & Increased \\
\hline mmu-miR-187-5p & 6.84 & 0.00173 & Increased \\
\hline mmu-miR-34a-5p & 6.67 & 0.02440 & Increased \\
\hline mmu-miR-744-3p & 6.55 & 0.00688 & Increased \\
\hline mmu-miR-224-3p & 6.45 & 0.01630 & Increased \\
\hline mmu-miR-365-2-5p & 6.20 & 0.00236 & Increased \\
\hline mmu-miR-455-5p & 6.09 & 0.03206 & Increased \\
\hline mmu-miR-122-3p & 5.84 & 0.02323 & Increased \\
\hline mmu-miR-145a-3p & 5.82 & 0.01150 & Increased \\
\hline mmu-miR-599 & 5.70 & 0.01257 & Increased \\
\hline mmu-miR-132-3p & 5.66 & 0.03740 & Increased \\
\hline mmu-miR-345-3p & 5.61 & 0.00401 & Increased \\
\hline mmu-miR-320-3p & 5.50 & 0.00795 & Increased \\
\hline mmu-miR-222-5p & 5.43 & 0.00712 & Increased \\
\hline mmu-miR-338-3p & 5.39 & 0.04550 & Increased \\
\hline mmu-miR-323-3p & 5.26 & 0.00462 & Increased \\
\hline
\end{tabular}


Table 1 Seventy-one differentially expressed $(p<0.05)$ circulating miRNAs in infected and control plasma samples (Continued)

\begin{tabular}{|c|c|c|c|}
\hline mmu-mir-92b-5p & 5.21 & 0.00752 & Increased \\
\hline mmu-miR-760-3p & 5.10 & 0.00402 & Increased \\
\hline mmu-miR-130a-5p & 4.94 & 0.00437 & Increased \\
\hline mmu-miR-212-3p & 4.91 & 0.04220 & Increased \\
\hline mmu-miR-490-5p & 4.89 & 0.00717 & Increased \\
\hline mmu-miR-7a-1-3p & 4.73 & 0.00781 & Increased \\
\hline mmu-miR-363-5p & 4.71 & 0.01730 & Increased \\
\hline mmu-miR-500-3p & 4.68 & 0.04449 & Increased \\
\hline mmu-miR-34b-3p & 4.42 & 0.03696 & Increased \\
\hline mmu-miR-188-3p & 4.39 & 0.01057 & Increased \\
\hline mmu-miR-709 & 4.34 & 0.01377 & Increased \\
\hline mmu-miR-30d-3p & 4.18 & 0.02385 & Increased \\
\hline$m m u-m i R-362-5 p$ & 4.09 & 0.01157 & Increased \\
\hline$m m u-m i R-448-3 p$ & 4.03 & 0.02179 & Increased \\
\hline mmu-miR-7a-5p & 3.94 & 0.02428 & Increased \\
\hline mmu-miR-196a-5p & 3.91 & 0.04891 & Increased \\
\hline mmu-miR-706 & 3.89 & 0.01287 & Increased \\
\hline mmu-miR-361-3p & 3.78 & 0.01351 & Increased \\
\hline mmu-miR-330-5p & 3.60 & 0.02304 & Increased \\
\hline mmu-miR-544-3p & 3.20 & 0.02653 & Increased \\
\hline mmu-miR-135a-1-3p & 3.07 & 0.04930 & Increased \\
\hline mmu-miR-710 & 2.95 & 0.04910 & Increased \\
\hline mmu-mir-181a-2-3p & 2.82 & 0.04925 & Increased \\
\hline mmu-miR-302a-3p & 0.16 & 0.01834 & decreased \\
\hline mmu-miR-495-3p & 0.16 & 0.02215 & decreased \\
\hline mmu-miR-454 & 0.16 & 0.04909 & decreased \\
\hline mmu-miR-688 & 0.05 & 0.02774 & decreased \\
\hline
\end{tabular}

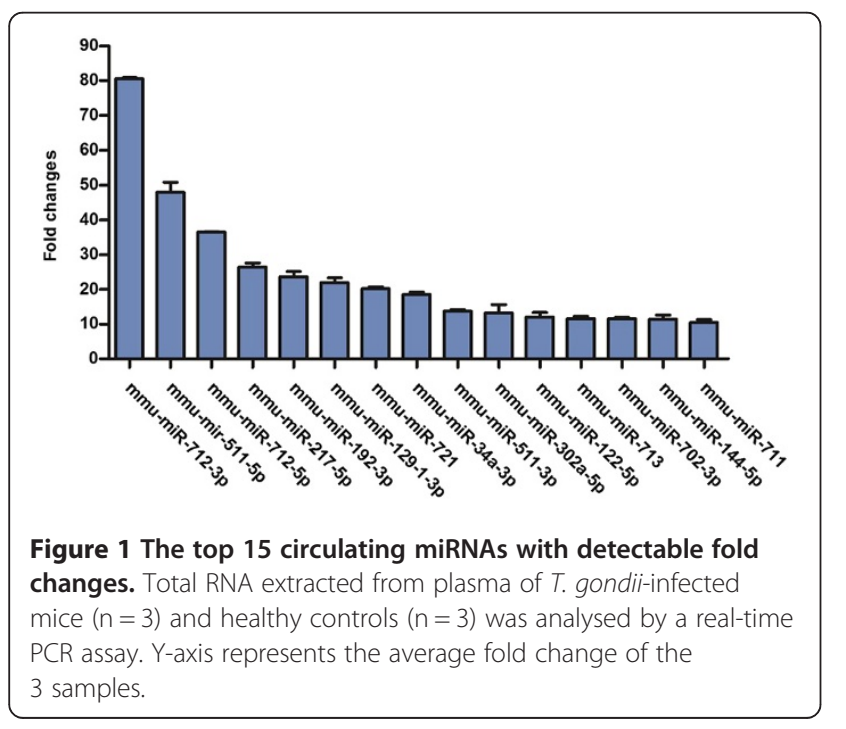

ME49 strain of T. gondii. We show that the three miRNAs were consistently up-regulated in the infected mice.

The reliability of the three miRNAs as markers for T. gondii infection was further scrutinised by ROC analysis, an analytical method frequently applied in previous studies. The AUC values of the tests of the three miRNAs in the infected mice were between 0.910 and 0.995 with sensitivities between $80 \%$ and $95 \%$, and a specificity of $100 \%$. Thus, the three miRNAs are likely to be specific responses to $T$. gondii infection. The miRNAs mmu-miR-712-5p and mmumiR-712-3p are the mature forms of mmu-miR-712, but the locations of their coding genes are still uncertain and their function unknown. Although both mmu-miR-712-5p and mmu-miR-712-3p were up-regulated in the plasma samples of T. gondii-infected mice, mmu-miR-712-3p was more significantly up-regulated. Therefore, we chose mmumiR-712-3p for further investigation. In previous studies, Son et al. reported that mmu-miR-712 derived from preribosomal RNA induced endothelial inflammation and 

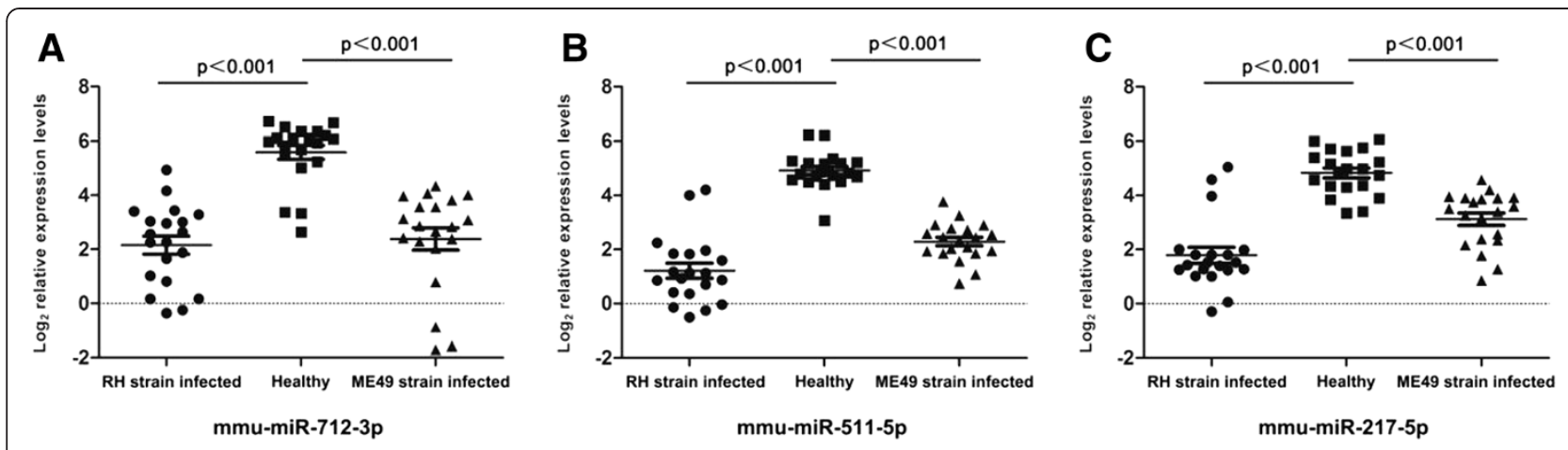

Figure 2 Validation of mmu-miR-712-3p, mmu-miR-511-5p and mmu-miR-217-5p in plasma samples $(\mathbf{n}=60)$. Scatter plots of plasma levels of mmu-miR-712-3p (A), mmu-miR-511-5p (B), and mmu-miR-217-5p (C) in mice infected with RH and ME49 strains of T. gondii ( $n=20$ each) and in healthy subjects $(n=20)$. Dark lines represent the mean values where appropriate. Expression levels of the miRNAs (log ${ }_{2}$ scale on the $y$-axis) were normalised to cel-miR-39.

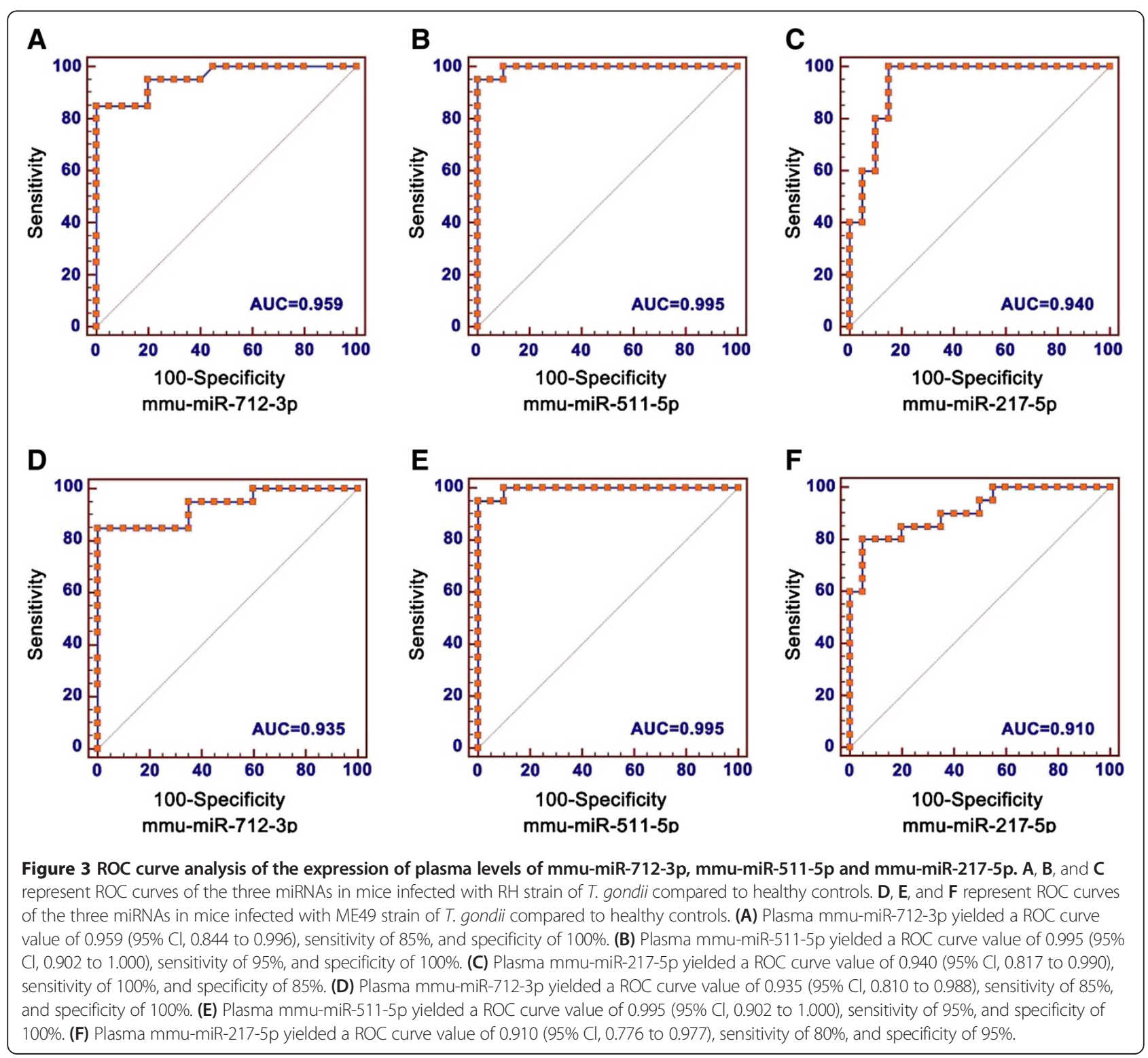



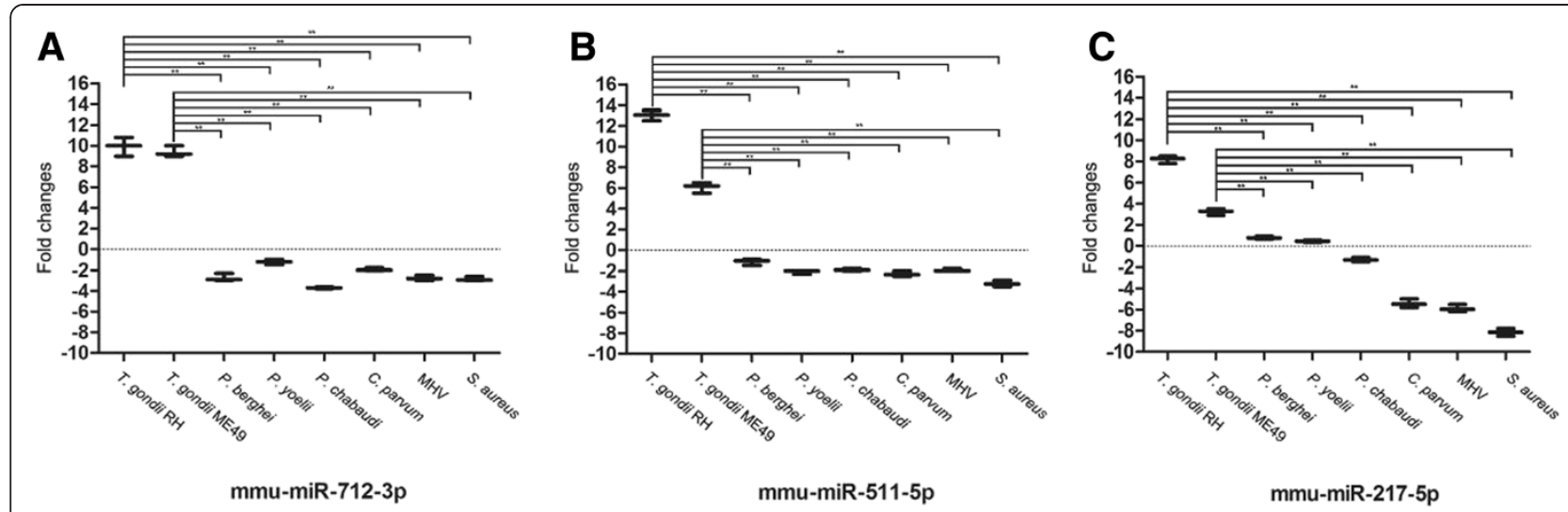

Figure 4 Relative levels of plasma mmu-miR-712-3p (A), mmu-miR-511-5p (B), and mmu-miR-217-5p (C) in mice infected with T. gondii RH and ME49 strains, P. berghei, P. yoelii, P. chabaudi, C. parvum, MHV, and S. aureus compared to healthy controls. The levels of the three miRNAs were specifically up-regulated in the plasma of mice infected with $T$. gondii compared to that in the plasma of mice infected by other organisms $(* * p<0.001)$.

atherosclerosis [33]. Mmu-miR-712-3p may be involved in vascular smooth muscle cell calcification by disrupting $\mathrm{Ca}^{2+}$ efflux proteins, which is not related to T. gondii infection [34]. Mmu-miR-511-5p was found significantly upregulated in the brain tissues of Angiostrongylus cantonensis (A. cantonensis) infected mice. This miRNA might play important role in the regulation of eosinophilic meningitis caused by A. cantonensis infection [35]. MiR-511 has been reported to positively regulate TLR4, as well as control macrophage production and activation [36]. Macrophages are important cells involved in the immune response to T. gondii infection and have been shown to inhibit parasite invasion and replication [37]. The elevated expression of mmu-miR-511-5p was likely a sign of host immune responses to $T$. gondii infection. However, further experiments are required to reveal the function of mmu-miR511-5p during T. gondii infections. Another over-expressed miRNA from the plasma of mice with $T$. gondii infection is mmu-miR-217-5p, which is known to be encoded by a gene located on chromosome 11. Previous studies indicated that miR-217 may act as a tumour suppressor, as suggested from a study on renal cell carcinoma and pancreatic ductal adenocarcinoma [38,39]. Up-regulation of miR-217 could decrease the expression of KRAS protein, thereby inhibiting tumour cell growth [39]. However, the function of mmumiR-217-5p in mice infected with $T$. gondii remains to be investigated.

To further determine that the up-regulation of $\mathrm{mmu}$ miR-712-3p, mmu-miR-511-5p and mmu-miR-217-5p was specific response to $T$. gondii infection, we compared the expression of these three miRNAs in mice infected with $T$. gondii to mice infected with $P$. berghei, P. yoelii, P. chabaudi, C. parvum, MHV, or S. aureus. The results showed that these three miRNAs were significantly up-regulated in mice infected with $T$. gondii but down-regulated in mice infected with other pathogens. The three murine Plasmodium species, C. parvum and $T$. gondii are all members of the phylum Apicomplexa, and they all cause severe infections in mice; however, the miRNA expression responses in the hosts were quite different. Furthermore, the expression of the three miRNAs was also found to be downregulated in mice infected with MHV and S. aureus. Thus, the data collectively suggest that the elevated responses of mmu-miR-712-3p, mmu-miR-511-5p and mmu-miR-217-5p in $T$. gondii infected mice were parasite-specific.

\section{Conclusion}

We report the evidence that three miRNAs, including mmu-miR-712-3p, mmu-miR-511-5p and mmu-miR217-5p, are significantly up-regulated in the plasma of mice after $T$. gondii infection, which may lead to the discovery of novel biomarkers for $T$. gondii infection.

\section{Competing interests}

The authors have declared that they have no competing interests.

\section{Authors' contributions}

BJ, QC and NJ conceived the design of experiment; BJ, ZC, XW, HL and JY carried out the experiments. The manuscript was drafted by BJ, QC and NJ. The final version was read and approved by all authors.

\section{Acknowledgement}

This study was supported by the provincial Natural Science Foundation of Jilin Province, China (20140101034JC) to Ning Jiang.

Received: 4 May 2014 Accepted: 6 August 2014

Published: 8 September 2014

\section{References}

1. Dubey J: Toxoplasma, Hammondia, Besnotia, Sarcosystis, and Other Tissue Cyst-Forming Coccidia of Man and Animals, Volume 3. New York: Academic Press; 1977:101-237. 
2. Dubey J, Beattie C: Toxoplasmosis of Animals and Man. Boca Raton: CRC Press; 1988:220.

3. Sun X, Lu H, Jia B, Chang Z, Peng S, Yin J, Chen Q, Jiang N: A comparative study of Toxoplasma gondii seroprevalence in three healthy Chinese populations detected using native and recombinant antigens. Parasit Vectors 2013, 6(1):241.

4. Fan CK, Lee LW, Liao CW, Huang YC, Lee YL, Chang YT, da Costa Ados S, Gil $\checkmark$, Chi LH, Nara T: Toxoplasma gondii infection: relationship between seroprevalence and risk factors among primary schoolchildren in the capital areas of Democratic Republic of Sao Tome and Principe, West Africa. Parasit Vectors 2012, 5:141

5. Sauer A, de la Torre A, Gomez-Marin J, Bourcier T, Garweg J, Speeg-Schatz C, Candolfi E: Prevention of retinochoroiditis in congenital toxoplasmosis: Europe versus South America. Pediatr Infect Dis J 2011, 30(7):601-603.

6. Bossi P, Caumes E, Astagneau P, Li TS, Paris L, Mengual X, Katlama C, Bricaire F: Epidemiologic characteristics of cerebral toxoplasmosis in 399 HIV-infected patients followed between 1983 and 1994. Rev Med Interne 1998, 19(5):313-317.

7. Gao XJ, Zhao ZJ, He ZH, Wang T, Yang TB, Chen XG, Shen JL, Wang Y, Lv FL, Hide G, Lun ZR: Toxoplasma gondii infection in pregnant women in China. Parasitology 2012, 139(2):139-147.

8. Karczewski G, Golab E: Diagnostic problems with congenital toxoplasmosis. Przegl Epidemiol 2011, 65(3):451-454.

9. Gilbert R: Treatment for congenital toxoplasmosis: finding out what works. Mem Inst Oswaldo Cruz 2009, 104(2):305-311.

10. Contini C: Clinical and diagnostic management of toxoplasmosis in the immunocompromised patient. Parassitologia 2008, 50(1-2):45-50.

11. Sensini A: Toxoplasma gondii infection in pregnancy: opportunities and pitfalls of serological diagnosis. Clin Microbiol Infect 2006, 12(6):504-512.

12. Lin MH, Chen TC, Kuo TT, Tseng CC, Tseng CP: Real-time PCR for quantitative detection of Toxoplasma gondii. J Clin Microbio/ 2000, 38(11):4121-4125.

13. Toth T, Sziller I, Papp Z: PCR detection of Toxoplasma gondii in human fetal tissues. Methods Mol Biol 1998, 92:195-202.

14. Guy EC, Pelloux H, Lappalainen M, Aspock H, Hassl A, Melby KK, Holberg-Pettersen $M$, Petersen E, Simon J, Ambroise-Thomas P: Interlaboratory comparison of polymerase chain reaction for the detection of Toxoplasma gondii DNA added to samples of amniotic fluid. Eur J Clin Microbiol Infect Dis 1996, 15(10):836-839.

15. Van Rooij E, Marshall WS, Olson EN: Toward microRNA-based therapeutics for heart disease: the sense in antisense. Circ Res 2008, 103(9):919-928.

16. Xu MJ, Zhou DH, Huang SY, Zhao FR, Nisbet AJ, Lin RQ, Song HQ, Zhu XQ: Comparative characterization of microRNA profiles of different genotypes of Toxoplasma gondii. Parasitology 2013, 140(9):1111-1118.

17. Wang J, Liu X, Jia B, Lu H, Peng S, Piao X, Hou N, Cai P, Yin J, Jiang N, Chen Q: A comparative study of small RNAs in Toxoplasma gondii of distinct genotypes. Parasit Vectors 2012, 5:186

18. Xu MJ, Zhou DH, Nisbet AJ, Huang SY, Fan YF, Zhu XQ: Characterization of mouse brain microRNAs after infection with cyst-forming Toxoplasma gondii. Parasit Vectors 2013, 6(1):154

19. Xiao J, Li Y, Prandovszky E, Karuppagounder SS, Talbot CC Jr, Dawson $\mathrm{VL}$, Dawson TM, Yolken RH: MicroRNA-132 dysregulation in Toxoplasma gondii infection has implications for dopamine signaling pathway. Neuroscience 2014, 268:128-138.

20. Cai $Y$, Chen $H$, Jin L, You Y, Shen J: STAT3-dependent transactivation of miRNA genes following Toxoplasma gondii infection in macrophage. Parasit Vectors 2013, 6:356.

21. Mitchell PS, Parkin RK, Kroh EM, Fritz BR, Wyman SK, Pogosova-Agadjanyan EL, Peterson A, Noteboom J, O'Briant KC, Allen A, Lin DW, Urban N, Drescher CW, Knudsen BS, Stirewalt DL, Gentleman R, Vessella RL, Nelson PS, Martin DB, Tewari M: Circulating microRNAs as stable blood-based markers for cancer detection. Proc Natl Acad Sci U S A 2008, 105(30):10513-10518.

22. Ng EK, Chong WW, Jin H, Lam EK, Shin WY, Yu J, Poon TC, Ng SS, Sung JJ: Differential expression of microRNAs in plasma of patients with colorectal cancer: a potential marker for colorectal cancer screening. Gut 2009, 58(10):1375-1381.

23. Silva J, Garcia V, Zaballos A, Provencio M, Lombardia L, Almonacid L, Garcia JM, Dominguez G, Pena C, Diaz R, Herrera M, Varela A, Bonilla F: Vesiclerelated microRNAs in plasma of nonsmall cell lung cancer patients and correlation with survival. Eur Respir J 2011, 37(3):617-623.
24. Morimura R, Komatsu S, Ichikawa D, Takeshita H, Tsujiura M, Nagata H, Konishi H, Shiozaki A, Ikoma H, Okamoto K, Ochiai T, Taniguchi H, Otsuji E: Novel diagnostic value of circulating miR-18a in plasma of patients with pancreatic cancer. Br J Cancer 2011, 105(11):1733-1740.

25. Shen J, Hruby GW, McKiernan JM, Gurvich I, Lipsky MJ, Benson MC, Santella RM: Dysregulation of circulating microRNAs and prediction of aggressive prostate cancer. Prostate 2012, 72(13):1469-1477.

26. Caggana M, Conroy JM, Pass KA: Rapid, efficient method for multiplex amplification from filter paper. Hum Mutat 1998, 11(5):404-409.

27. Homan WL, Vercammen $\mathrm{M}$, De Braekeleer J, Verschueren $\mathrm{H}$ : Identification of a 200 - to 300 -fold repetitive 529 bp DNA fragment in Toxoplasma gondii, and its use for diagnostic and quantitative PCR. Int J Parasito/ 2000, 30(1):69-75.

28. Ozer J, Ratner M, Shaw M, Bailey W, Schomaker S: The current state of serum biomarkers of hepatotoxicity. Toxicology 2008, 245(3):194-205.

29. Amacher DE, Adler R, Herath A, Townsend RR: Use of proteomic methods to identify serum biomarkers associated with rat liver toxicity or hypertrophy. Clin Chem 2005, 51(10):1796-1803.

30. Fichtlscherer S, De Rosa S, Fox H, Schwietz T, Fischer A, Liebetrau C, Weber M, Hamm CW, Roxe T, Muller-Ardogan M, Bonauer A, Zeiher AM, Dimmeler S: Circulating microRNAs in patients with coronary artery disease. Circ Res 2010, 107(5):677-684.

31. Lawrie CH, Gal S, Dunlop HM, Pushkaran B, Liggins AP, Pulford K, Banham AH, Pezzella F, Boultwood J, Wainscoat JS, Hatton CS, Harris AL: Detection of elevated levels of tumour-associated microRNAs in serum of patients with diffuse large B-cell lymphoma. Br J Haematol 2008, 141(5):672-675

32. McDonald JS, Milosevic D, Reddi HV, Grebe SK, Algeciras-Schimnich A: Analysis of circulating microRNA: preanalytical and analytical challenges. Clin Chem 2011, 57(6):833-840.

33. Son DJ, Kumar S, Takabe W, Kim CW, Ni CW, Alberts-Grill N, Jang IH, Kim S, Kim W, Won Kang S, Baker AH, Woong Seo J, Ferrara KW, Jo H: The atypical mechanosensitive microRNA-712 derived from pre-ribosomal RNA induces endothelial inflammation and atherosclerosis. Nat Commun 2013, 4:3000.

34. Gui T, Zhou G, Sun Y, Shimokado A, Itoh S, Oikawa K, Muragaki Y: MicroRNAs that target $\mathrm{Ca}(2+)$ transporters are involved in vascular smooth muscle cell calcification. Lab Invest 2012, 92(9):1250-1259.

35. Yu L, Liao Q, Zeng X, Lv Z, Zheng H, Zhao Y, Sun X, Wu Z: MicroRNA expressions associated with eosinophilic meningitis caused by Angiostrongylus cantonensis infection in a mouse model. Eur J Clin Microbiol Infect Dis 2014, 33(8):1457-1465.

36. Tserel L, Runnel T, Kisand K, Pihlap M, Bakhoff L, Kolde R, Peterson H, Vilo J, Peterson P, Rebane A: MicroRNA expression profiles of human blood monocyte-derived dendritic cells and macrophages reveal miR-511 as putative positive regulator of Toll-like receptor 4. J Biol Chem 2011, 286(30):26487-26495.

37. Hou B, Benson A, Kuzmich L, DeFranco AL, Yarovinsky F: Critical coordination of innate immune defense against Toxoplasma gondii by dendritic cells responding via their Toll-like receptors. Proc Natl Acad Sci U S A 2011, 108(1):278-283.

38. Li H, Zhao J, Zhang JW, Huang QY, Huang JZ, Chi LS, Tang HJ, Liu GQ, Zhu DJ, Ma WM: MicroRNA-217, down-regulated in clear cell renal cell carcinoma and associated with lower survival, suppresses cell proliferation and migration. Neoplasma 2013, 60(5):511-515.

39. Zhao WG, Yu SN, Lu ZH, Ma YH, Gu YM, Chen J: The miR-217 microRNA functions as a potential tumor suppressor in pancreatic ductal adenocarcinoma by targeting KRAS. Carcinogenesis 2010, 31(10):1726-1733.

doi:10.1186/1756-3305-7-433

Cite this article as: Jia et al:: Plasma microRNAs are promising novel biomarkers for the early detection of Toxoplasma gondii infection. Parasites \& Vectors 2014 7:433 